\title{
High- and low-resolution spectroscopic observations of the peculiar planetary-nebula HD $149427(=\mathrm{PC} 11)^{\star, \star \star}$
}

\author{
C. B. Pereira ${ }^{1}$, N. O. Baella ${ }^{1}$, S. Daflon ${ }^{1}$, and L. F. Miranda ${ }^{2}$ \\ 1 Observatório Nacional-MCT, Rua José Cristino, 77. CEP 20921-400 São Cristóvão, Rio de Janeiro-RJ, Brazil \\ e-mail: [claudio; baella; daflon]@on.br \\ 2 Instituto de Astrofísica de Andalucía, CSIC, Apdo. 3004, 18080 Granada, Spain \\ e-mail: lfm@iaa.es
}

Received 8 April 2009 / Accepted 5 August 2009

ABSTRACT

\begin{abstract}
Aims. We discuss the status of the A-type component of the binary central star in the peculiar planetary nebula HD 149427. Methods. We acquired low and high spectral resolution optical spectroscopy for the range 4500-9000 ̊.

Results. The analysis of the high resolution spectrum shows that the A-type star has an effective temperature of $T_{\text {eff }}=8700 \pm 150 \mathrm{~K}$ and a surface gravity of $\log g=2.3 \pm 0.4$, corresponding to a spectral type A3-A4 II. These parameters infer a luminosity estimate of $\log \left(L / L_{\odot}\right)=3.69 \pm 0.32$, implying a distance of $10 \pm 0.6 \mathrm{kpc}$. The A-type star is slightly metal-poor; barium and strontium, two key elements for monitoring the s-process nucleosynthesis, are not enriched; nitrogen is enriched relative to Sun; while carbon and oxygen are underabundant. The photospheric abundance of the A-type giant star in HD 149427 is compared with the abundance in other types of stars at different evolutionary stages.
\end{abstract}

Key words. planetary nebulae: individual: HD 149427 - stars: abundances - stars: fundamental parameters

\section{Introduction}

Among the 1820 objects classified at least once as planetary nebulae (PNe) in the Strasbourg-ESO Catalogue of Planetary Nebulae (Acker et al. 1992), there is a very small number of PNe known to contain close binary nuclei. Bond (2000) lists only 16 objects. These objects are relevant because they may provide direct evidence of the occurrence of common envelope evolution in these binary systems. Some of them have been discovered because of their photometric variability (either due to stellar eclipses or reflection effects). Miszalski et al. (2008) identified three new binary central stars in PNe using photometry, although no further information about the nature of the central star was attainable using spectroscopy.

In some central stars of $\mathrm{PNe}$, the companion is resolved and its their spectroscopic characteristics can be investigated. Méndez (1989) provided such a list and performed a radial velocity survey of binary central stars of PNe, where the several types of companion of the hot star are listed according to the methods of discovery. His Table 1 contains a list of central stars of PNe (hereafter CSPN). The objects in this list represent what had been previously classified as "peculiar PNe" according to Lutz (1977). In particular, Lutz (1977) defined peculiar PNe, to be objects that have nebular emission lines in combination with an absorption spectrum of a cool central star (of spectral types from A to K). Since A to K stars are not hot enough to ionize the nebulae, these peculiar PNe should be binary systems consisting

$\star$ Based on observations made with the $1.52 \mathrm{~m}$ and $2.2 \mathrm{~m}$ telescope at the European Southern Observatory (La Silla, Chile).

$\star \star$ Present address: Departamento de Física Aplicada, Facultade de Ciencias, Universidade de Vigo, 36310 Vigo, Spain. of a faint hot star (responsible for the ionization of the nebulae) plus a bright $\mathrm{A}$ to $\mathrm{K}$ star that is the source of the absorption spectrum. Nowadays, it is known that some objects in the list of Lutz's (1977) belong to a small subsample of symbiotic stars called yellow symbiotics (Schmid \& Nussbaumer 1993). Spectroscopically detected CSPN with spectral types G and K in these peculiar PNe have already been studied in the literature. The most representative objects of this class are indeed Abell 35 and Lotr 5 (Thevenin \& Jasniewicz 1997). WeBo1 (Bond et al. 2003) and Me 1-1 (Shen et al. 2004; Pereira et al. 2008) can be included in this very short list. de Marco (2009) discusses and reviews the binary hypothesis of CSPN, Tables 2 and 3 of her paper presents a list of all known binary CSPNe.

In this work, we analyze low- and high-resolution spectra of the peculiar PN HD 149427. HD 149427 (=Hen 2-172= PC 11) was first noticed to be a peculiar object by Webster (1966) on the basis of it strong continuum the absorption Ca II line accompanied by nebular emission lines. She also noted that the [O III] $\lambda 4363$ emission line was very strong (see also Sanduleak \& Stephenson 1972) and realized that the spectrum of HD 149427 was very similar to the $\mathrm{D}^{\prime}$-type symbiotic object HD 330036. The optical and ultraviolet emission line spectra of HD 149427 were investigated by Gutiérrez-Moreno \& Moreno (1998) and Parthasarathy et al. (2000), respectively. In these previous studies, the authors determined the chemical composition of the nebula, the temperature of the hot star and discussed the nature of HD 149427 based on its ultraviolet spectrum. Our data allow us to determine some physical properties of both the hot star, and the ionized nebula and to derive the atmospheric parameters as well as the chemical composition of the cool star in the system. 

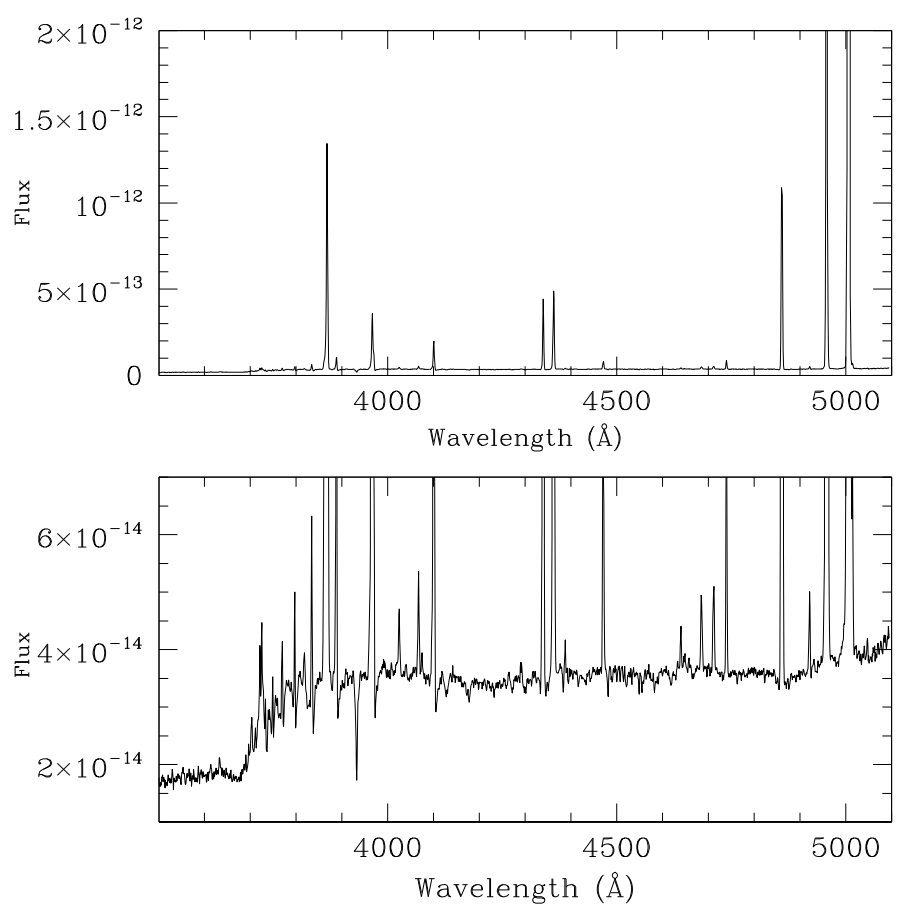

Fig. 1. Optical spectrum of HD 149427 between $3100 \AA$ and $5100 \AA$. Notice the strength of the [O III] $\lambda 4363$ in the upper panel. In the lower panel the scale is enlarged to better show some absorption lines of the A-F star. Also notice the the Ca II absorption line around 3933, $67 \AA$ as first noticed by Webster (1966). The units of flux are erg/ $/ \mathrm{cm}^{2} / \mathrm{s} / \AA$.

\section{Observations}

\subsection{Low-resolution spectroscopy}

The low-resolution spectroscopic observations were performed using a Boller \& Chivens spectrograph at the Cassegrain focus of the ESO $1.52 \mathrm{~m}$ telescope in La Silla (Chile). A UV-flooded thinned Loral Lesser CCD \#39 $(2048 \times 2048,15 \mu \mathrm{m} /$ pixel $)$ was used as detector providing a high quantum efficiency in both the blue and in the UV. Two instrumental setups were employed. The first setup used the grating \#23 with $600 \mathrm{l} / \mathrm{mm}$, providing a spectral resolution of $4.6 \AA$ in the range 3500-7500 $\AA$ (May 21, 1997). The second setup used of the grating \#31 with $1200 \mathrm{l} / \mathrm{mm}$, resulting in a resolution of $1.9 \AA$ in the range 3100-5100 (May 22, 1997).

The data were reduced to linear scale, i.e., wavelength versus flux, using IRAF. We followed the standard procedure consisting of bias subtraction, flat-field normalization, and wavelength calibration bt using a He-Ar lamp. Counts were corrected for atmospheric extinction and calibrated for instrumental chromatic response by observations of standard stars from Oke (1974) and Hamuy et al. (1994). In the linearized spectra, the line fluxes were measured with the splot task and blends were resolved using the deblend option. Figure 1 shows the reduced spectrum of HD 149427 in the spectral range between $3100 \AA$ and $5100 \AA$. We estimate the errors in the fluxes to be about $10 \%$ for stronger lines (line fluxes similar to $\mathrm{H} \beta$ on the scale of $\mathrm{H} \beta=100$ ).

\subsection{High-resolution spectroscopy}

The high-resolution spectrum of HD 149427 analyzed in this work was obtained at the $2.2 \mathrm{~m}$ ESO telescope on April 3, 2007 with the FEROS (Fiberfed Extended Range Optical Spectrograph) echelle spectrograph (Kaufer et al. 1999). The
Table 1. Emission line fluxes in units of $\mathrm{H} \beta=100$ of some Balmer and [O III] emission lines.

\begin{tabular}{lc}
\hline \hline Ion & $F(\lambda)$ \\
\hline $\mathrm{H} \delta$ & 15.0 \\
$\mathrm{H} \gamma$ & 35.0 \\
{$[\mathrm{O}$ III] $] 4363$} & 43.0 \\
{$[\mathrm{O}$ III $] \lambda 5007$} & 1438.0 \\
\hline
\end{tabular}

FEROS spectral resolving power is $R=48000$ corresponding to 2.2 pixels of $15 \mu \mathrm{m}$ and the wavelength coverage is from $4000 \AA$ to $9200 \AA$. The nominal $S / N$ ratio was evaluated by the measurement of the rms flux fluctuation in selected continuum windows, and a typical value achieved was $S / N \approx 100$, after $2 \times 3600 \mathrm{~s}$ of integration time. Data were reduced with IRAF following the procedures mentioned above (see Sect. 2.1).

\section{Analysis and results}

\subsection{Low-resolution spectroscopy}

The low-resolution spectrum of HD 149427 (see Fig. 1) exhibts nebular emission lines (e.g., [O III], [N II], Balmer lines), on the continuum of an A-F type star. The oxygen line [O III] $\lambda 4363$ is stronger than $\mathrm{H} \gamma$, which is indicative of high electron density (see below and Pereira \& Miranda 2005). A strong Ca II absorption and several other absorptions in the spectrum between $3500 \AA$ and $5100 \AA$ can also been identified (see below). Table 1 shows the emission line fluxes of some Balmer and [O III] emission lines in units of $\mathrm{H} \beta=100.0$. The low-resolution emission spectrum of HD 149427 was analyzed by Gutiérrez-Moreno \& Moreno (1998) and we note that the strengths of the emission line did not significantly change between their observations and ours. In this work we used our emission-line fluxes to obtain the reddening in the direction to HD 149427 in order to better constraint its distance and the luminosity of both the A-type star and the hot star in the system (Sect. 4.1). We also used the low resolution spectrum of HD 149427 to compare it with other PNe and symbiotic stars (Sect. 5).

The extinction parameter was derived from the $\mathrm{H} \gamma / \mathrm{H} \beta$ and $\mathrm{H} \delta / \mathrm{H} \beta$ line ratios. We assumed that the reddening law can be represented by the standard interstellar extinction curve $f(\lambda)$ (Seaton 1979). For HI, we assumed that the emission is produced in case B conditions (Hummer \& Storey 1987) with a mean electron temperature $T_{\mathrm{e}}=20000 \mathrm{~K}$ and and a mean electron density $N_{\mathrm{e}}=10^{5} \mathrm{~cm}^{-3}$ (see Gutiérrez-Moreno \& Moreno 1998). Using this procedure and on the basis of the observed line ratios, we obtain $E(B-V)=0.68$, which is similar to the value derived by Tylenda et al. (1992). A previous determination of interstellar reddening was provided by Parthasarathy et al. (2000) based on the absorption dip at $2200 \AA$. The authors found $E(B-V)=0.4 \pm 0.2$ which is in marginal agreement with ours, probably because of the low quality of the UV spectra in the $2200 \AA$ A region.

\subsection{High-resolution spectroscopy}

\subsubsection{Physical properties: effective temperature and gravity}

Figures 2 and 3 show the high-resolution spectrum of HD 149427 and of the A1 III star HD 75063 (Royer et al. 2002), in the regions around 4500-4600 $\AA$ and 6130-6180 $\AA$, respectively. As we shall show, HD 149427 is also a giant with a 


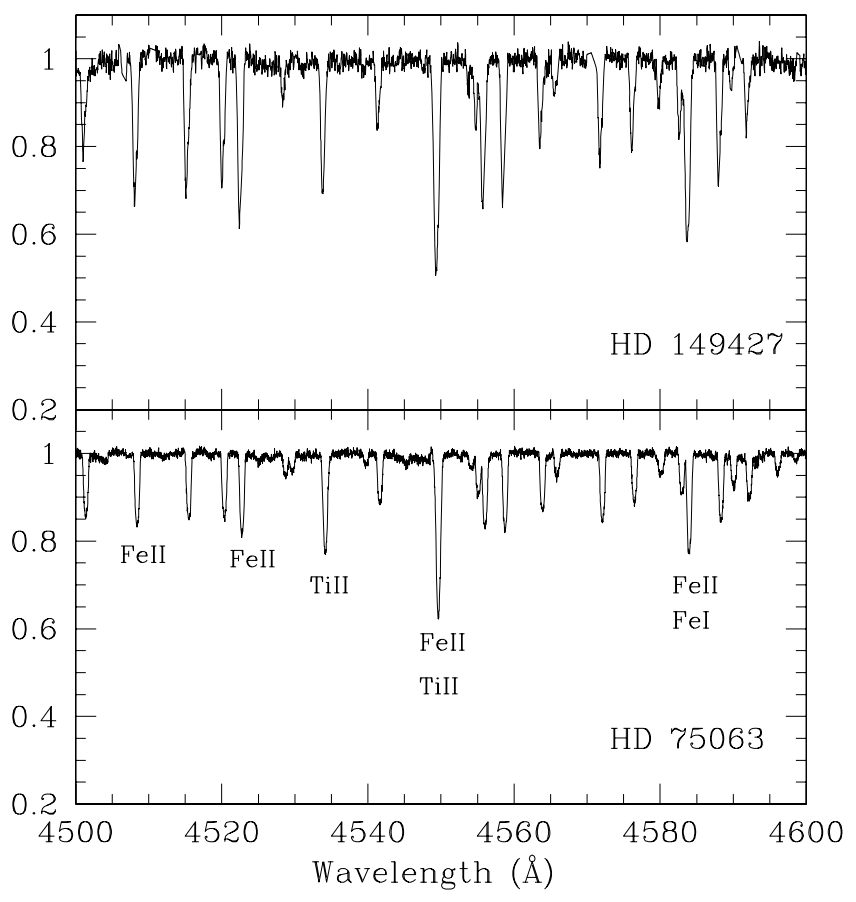

Fig. 2. Observed absorption spectrum of HD 149427 and HD 75063 between $4500 \AA$ and $4600 \AA$.

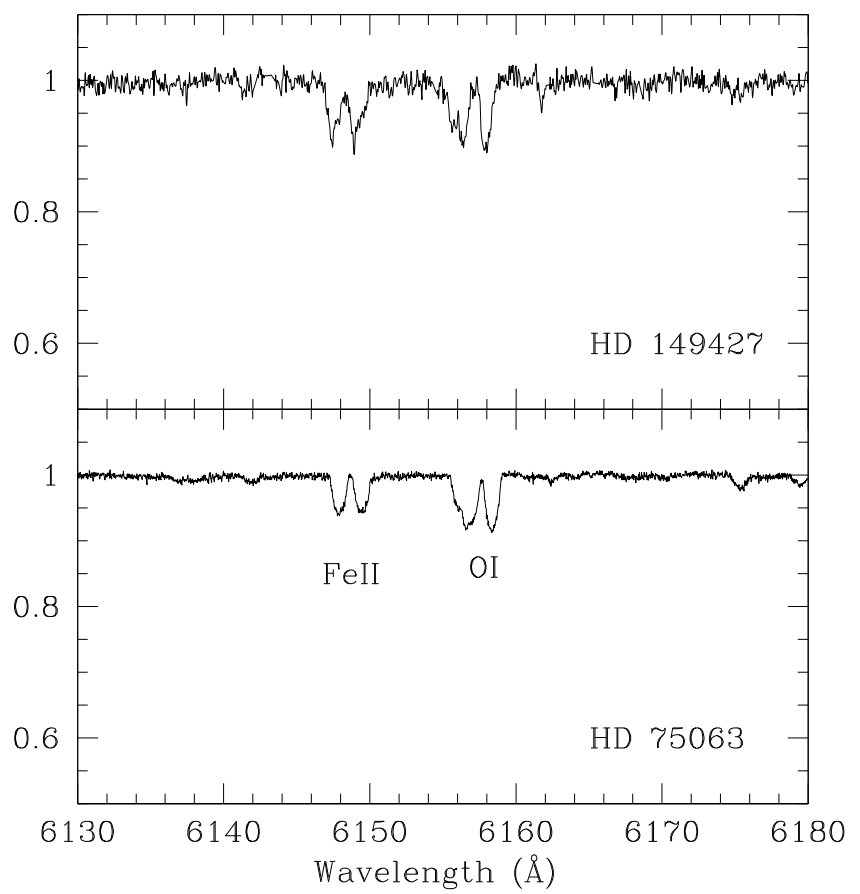

Fig. 3. Observed absorption spectrum of HD 149427 and of HD 75063 between $6130 \AA$ and $6180 \AA$.

spectral type A3-A4. Several absorption lines of Fe I and Fe II are identified as well as Ti II, Cr II, Mg I, Sr II, and Ba II absorption lines. In the red, some transitions due to C I and N I are identified. In Table 2, we list the Fe I and Fe II lines employed in the analysis in addition to the lower excitation potential, $\chi(\mathrm{eV})$, of the transitions, the $g f$-values, and the measurements of the equivalent widths. The last set of measurements were obtained by fitting Gaussian profiles to the observed ones. The references for the $g f$-values are given in the fifth column of Table 2. Table 3 provides a line list of the other elements.
The determination of stellar atmospheric parameters, effective temperature $\left(T_{\text {eff }}\right)$, surface gravity $(\log g)$, microturbulence $(\xi)$, and $[\mathrm{Fe} / \mathrm{H}]$ (throughout, we use the notation $[\mathrm{X} / \mathrm{H}]=$ $\log (N(\mathrm{X}) / N(\mathrm{H}))_{\star}-\log \left(N(\mathrm{X}) / N(\mathrm{H})_{\odot}\right)$ are prerequisites for the determination of photospheric abundances. The atmospheric parameters were determined by assuming local-thermodynamicequilibrium (hereafter LTE) model-atmospheres of Kurucz (1993) using the spectral analysis code MOOG (Sneden 1973).

The solution of the excitation equilibrium used to derive the temperature $\left(T_{\text {eff }}\right)$ was defined by zero slope of the trend between the Fe I abundances and the excitation potential of the measured lines. The microturbulent velocity $\left(V_{\mathrm{t}}\right)$ was determined by constraining the abundance determined from individual Fe I lines to show no dependence on $W_{\lambda} / \lambda$. The solution thus found is unique, depending only on the set of Fe I, II lines and the atmospheric model employed, and yields as a by-product the metallicity of the star $[\mathrm{Fe} / \mathrm{H}]$.

The result for the abundance derived from each line is given in the seventh column and the ratio $[\mathrm{Fe} / \mathrm{H}]$ in the last column of Table 2. The gravity was determined from the ionization equlibrium of the Fe I and Fe II to yield the same iron abundance at the selected effective temperature. The final adopted atmospheric parameters are $T_{\mathrm{eff}}=8700 \pm 150 \mathrm{~K}, \log g=2.3 \pm$ $0.4 \mathrm{~cm} / \mathrm{s}^{2}, V_{\mathrm{t}}=5.8 \pm 0.5 \mathrm{~km} \mathrm{~s}^{-1}$ and $[\mathrm{Fe} / \mathrm{H}]=-0.20 \pm 0.17$. The star's projected rotation velocity was also derived and we obtain $v \sin i=30 \pm 5 \mathrm{~km} \mathrm{~s}^{-1}$.

To check our analysis of HD 149427, we also analyzed the spectrum of the A7-A8 giant HD 19993 with the same method. The derived stellar parameters for HD 19993 are $T_{\text {eff }}=7600 \pm$ $150 \mathrm{~K}, \log g=2.7 \pm 0.3 \mathrm{~cm} / \mathrm{s}^{2}, V_{\mathrm{t}}=2.5 \pm 0.2 \mathrm{~km} \mathrm{~s}^{-1}$, and $[\mathrm{Fe} / \mathrm{H}]=-0.24 \pm 0.11$, in good agreement with the previous determinations of Miroshnichenko et al. (2003) of $T_{\text {eff }}=7500 \pm$ $200 \mathrm{~K}, \log g=2.7 \pm 0.2 \mathrm{~cm} / \mathrm{s}^{2}, V_{\mathrm{t}}=2.9 \pm 0.2 \mathrm{~km} \mathrm{~s}^{-1}$ and $[\mathrm{Fe} / \mathrm{H}]=-0.34 \pm 0.12$.

The atmosphere parameters $T_{\text {eff }}$ and $\log g$ can also be determined independently from the analysis of hydrogen absorption line profiles, obtained from the comparison between model $\mathrm{H} \gamma$ line wings and observed profiles (Daflon et al. 2004). The value of $T_{\text {eff }}$ was varied accross the range $8000-11000 \mathrm{~K}$, in steps of $1000 \mathrm{~K}$ and the theoretical profiles were interpolated from a grid computed for LTE with ATLAS9, allowing the $\log g$ to vary in order to reproduce the observed profile. The central region $(\sim 3 \AA)$ of the $\mathrm{H} \gamma$ profiles were not considered in the fits, because of non-LTE effects that can affect mainly the line cores. The best fit model was chosen based on a $\chi^{2}$ minimization of the differences between the observed and the theoretical profiles. In Fig. 4, we illustrate the best fit theoretical profile (solid green line) and the observed spectrum (solid black line) in the region of $\mathrm{H} \gamma$ for the star $\mathrm{HD} 149427$, for $T_{\text {eff }}=9000 \mathrm{~K}$ and $\log g=2.25$, which agree very well with the adopted parameters for this star. The profiles computed for both $T_{\mathrm{eff}}=9000 \mathrm{~K}$ and $\log g=2.00,2.50$, and 4.00 are also shown in red lines. The discrepancy between the observed spectrum and the model profile calculated for $\log g=4.00$ reinforces our result that HD 149427 is very likely to be a star that has evolved away from the main sequence.

Previous determination of the atmospheric parameters (temperature and gravity) for the cool star in HD 149427 was carried out by Parthasarathy et al. (2000). The authors obtained a temperature around $7500 \mathrm{~K}$ (an early F-type star) and $\log g=$ 4.5 dex, although this value for $\log g$ should be treated with caution. Low-resolution spectroscopy is not accurate enough to determine the luminosity class and, hence, $\log g$. For lowresolution spectroscopy, the most useful lines for investigating 
Table 2. Observed Fe I and Fe II absorption lines in HD 149427.

\begin{tabular}{|c|c|c|c|c|c|c|c|}
\hline Element & $\lambda(\AA)$ & $\chi(\mathrm{eV})$ & $\log g f$ & Ref. & $W_{\lambda}(\mathrm{m} \AA)$ & $\log \epsilon$ & {$[\mathrm{Fe} / \mathrm{H}]$} \\
\hline \multirow{22}{*}{ Fe I ...... } & 3922.91 & 0.05 & -1.626 & m1995 & 98 & 7.29 & \\
\hline & 3930.30 & 0.09 & -1.490 & $\mathrm{n} 2001$ & 112 & 7.27 & \\
\hline & 4132.06 & 1.61 & -0.670 & s2004 & 86 & 7.26 & \\
\hline & 4181.76 & 2.83 & -0.180 & s2004 & 49 & 7.24 & \\
\hline & 4202.03 & 1.48 & -0.700 & s2004 & 100 & 7.30 & \\
\hline & 4222.22 & 2.45 & -0.914 & m1995 & 24 & 7.36 & \\
\hline & 4227.44 & 3.33 & 0.266 & m1995 & 87 & 7.46 & \\
\hline & 4260.47 & 2.40 & -0.020 & s2004 & 125 & 7.41 & \\
\hline & 4282.41 & 2.18 & -0.779 & m1995 & 48 & 7.39 & \\
\hline & 4404.75 & 1.56 & -0.140 & mcw & 129 & 6.98 & \\
\hline & 4466.56 & 2.83 & -0.600 & s2004 & 30 & 7.39 & \\
\hline & 4918.99 & 2.87 & -0.340 & s2004 & 55 & 7.46 & \\
\hline & 5232.94 & 2.94 & -0.080 & s2004 & 86 & 7.51 & \\
\hline & 5328.04 & 0.92 & -1.470 & s2004 & 69 & 7.40 & \\
\hline & 5371.49 & 0.96 & -1.650 & s2004 & 25 & 7.07 & \\
\hline & 5383.37 & 4.31 & 0.640 & s2004 & 27 & 7.05 & \\
\hline & 5393.18 & 3.24 & -0.716 & m1995 & 20 & 7.56 & \\
\hline & 5397.14 & 0.92 & -1.990 & s2004 & 33 & 7.52 & \\
\hline & 5405.77 & 0.99 & -1.850 & s2004 & 30 & 7.38 & \\
\hline & 5415.21 & 4.39 & 0.640 & a2007 & 24 & 7.05 & \\
\hline & & & & & & 7.32 & -0.20 \\
\hline & & & & & & \pm 0.17 & \\
\hline \multirow[t]{21}{*}{ Fe II ..... } & 4296.58 & 2.70 & -3.010 & v93 & 148 & 7.33 & \\
\hline & 4369.41 & 2.78 & -3.670 & v93 & 69 & 7.45 & \\
\hline & 4472.93 & 2.84 & -3.430 & 186 & 59 & 7.16 & \\
\hline & 4489.18 & 2.84 & -2.970 & n1996 & 133 & 7.26 & \\
\hline & 4576.34 & 2.84 & -2.970 & n1996 & 140 & 7.31 & \\
\hline & 4582.83 & 2.84 & -3.100 & n1996 & 105 & 7.19 & \\
\hline & 4620.52 & 2.83 & -3.287 & m1995 & 96 & 7.31 & \\
\hline & 4635.32 & 5.95 & -1.650 & n1996 & 64 & 7.38 & \\
\hline & 4666.75 & 2.83 & -3.310 & 196 & 92 & 7.30 & \\
\hline & 5284.10 & 2.89 & -3.010 & 196 & 132 & 7.33 & \\
\hline & 5325.56 & 3.22 & -3.170 & 196 & 79 & 7.33 & \\
\hline & 5414.05 & 3.22 & -3.620 & 196 & 22 & 7.09 & \\
\hline & 5425.25 & 3.20 & -3.210 & 196 & 65 & 7.23 & \\
\hline & 5534.83 & 3.25 & -2.770 & 196 & 128 & 7.31 & \\
\hline & 5991.37 & 3.15 & -3.560 & 196 & 39 & 7.29 & \\
\hline & 6149.25 & 3.89 & -2.720 & 196 & 88 & 7.42 & \\
\hline & 6247.55 & 3.89 & -2.340 & 196 & 138 & 7.42 & \\
\hline & 6416.92 & 3.89 & -2.680 & 196 & 78 & 7.31 & \\
\hline & 6432.68 & 2.89 & -3.580 & 196 & 67 & 7.44 & \\
\hline & & & & & & 7.31 & -0.21 \\
\hline & & & & & & \pm 0.09 & \\
\hline
\end{tabular}

References: a2007: Aoki et al. (2007); 196: Lambert et al. (1996); m1995: McWilliam et al. (1995); n1996: Norris et al. (1996); n2001: Norris et al. (2001); s2004: Sivarani et al. (2004); v93: Venn (1993).

the luminosity class, are in the red (Pereira \& Miranda 2007). In addition, a comparison of the UV low-resolution spectrum of HD 149427 with that of the F0V HD 58936 star (Fig. 3 of Parthasarathy et al. 2000) shows that there are several regions in the spectrum of HD 149427 that are not matched well by the comparison star.

Cool CSPN with spectral types earlier than F have already been reported in the literature but their analyses are scarce. Table 4 of de Marco (2009) lists 6 CSPN with spectral types B and A. Méndez (1978) analyzed three A-type CSPN (NGC 2346, NGC 3132, and Hen 2-36) and obtained their temperatures and gravities but not their photospheric abundances. We note that the cool central star of HD 149427 is similar to Hen 2-36, which has a spectral type A2 III.

\subsubsection{Abundance analysis}

Elemental abundances of the A-type star of HD 149427 were determined with the LTE model atmosphere techniques already described by Pereira (2004). In brief, equivalent widths are calculated by integrating through a model atmosphere and are compared with the observed equivalent widths. The calculation is repeated, changing the abundance of the element in question, until a close match is achieved. The line-synthesis code MOOG was used to carry out the calculations. The results are given in Table 3, where we list the lines employed for each species, the abundances derived from each line, and the ratio $[\mathrm{X} / \mathrm{H}]$.

The internal scatter in the derived abundances of each element with more than two lines is also given in Table 3 . The uncertainties in the derived abundances are dominated by the 
C. B. Pereira et al.: Spectroscopic analysis of the peculiar planetary nebula HD 149427

Table 3. Other absorption lines studied and their respective abundances.

\begin{tabular}{|c|c|c|c|c|c|c|c|}
\hline$\lambda(\AA)$ & Species & $\chi(\mathrm{eV})$ & $\log -g f$ & Ref. & $W_{\lambda}(\mathrm{m} \AA)$ & $\log \epsilon$ & {$[\mathrm{X} / \mathrm{H}]$} \\
\hline 9078.57 & $\mathrm{C}_{\mathrm{I}}$ & 7.48 & -0.58 & v93 & 305 & 7.74 & \\
\hline 9094.89 & C I & 7.49 & 0.16 & v93 & 407 & 7.64 & \\
\hline \multirow[t]{2}{*}{9111.85} & C I & 7.49 & -0.29 & v93 & 384 & 7.96 & \\
\hline & & & & & & $\begin{array}{c}7.78 \\
\pm 0.16\end{array}$ & -0.74 \\
\hline 8683.40 & $\mathrm{~N}_{\mathrm{I}}$ & 10.34 & -0.05 & v93 & 223 & 8.28 & \\
\hline 8686.15 & N I & 10.33 & -0.35 & v93 & 115 & 8.20 & \\
\hline 8703.24 & N I & 10.34 & -0.34 & v93 & 155 & 8.07 & \\
\hline 8711.69 & N I & 10.34 & -0.24 & v93 & 150 & 7.94 & \\
\hline \multirow[t]{2}{*}{8718.82} & N I & 10.34 & -0.35 & v93 & 204 & 8.49 & \\
\hline & & & & & & 8.19 & +0.27 \\
\hline 615599 & $\mathrm{OI}_{\mathrm{I}}$ & 10.74 & -0.670 & v93 & 65 & \pm 0.19 & \\
\hline & & & & Vy3 & 65 & 8.68 & \\
\hline 6156.78 & O I & 10.74 & -0.450 & v93 & 73 & 8.55 & \\
\hline \multirow[t]{3}{*}{6158.19} & O I & 10.74 & -0.310 & v93 & 93 & 8.61 & \\
\hline & & & & & & 8.61 & -0.22 \\
\hline & & & & & & \pm 0.06 & \\
\hline 4057.51 & $\operatorname{Mg} I$ & 4.35 & -0.890 & n1996 & 45 & 7.52 & \\
\hline 4167.28 & Mg I & 4.34 & -0.710 & n1996 & 55 & 7.44 & \\
\hline 4702.99 & Mg I & 4.34 & -0.520 & a2007 & 62 & 7.31 & \\
\hline 5167.32 & Mg I & 2.71 & -0.860 & v93 & 179 & 7.42 & \\
\hline \multirow[t]{3}{*}{5528.42} & Mg I & 4.34 & -0.490 & a2007 & 75 & 7.41 & \\
\hline & & & & & & 7.42 & -0.16 \\
\hline & & & & & & \pm 0.08 & \\
\hline 6347.11 & Si II & 8.12 & 0.225 & wsm & 201 & 7.15 & \\
\hline \multirow{2}{*}{6371.37} & Si II & 8.12 & -0.074 & wsm & 129 & 6.88 & \\
\hline & & & & & & 7.02 & -0.53 \\
\hline \multirow[t]{2}{*}{4226.74} & $\mathrm{Ca} I$ & 0.00 & 0.240 & n1996 & 130 & 5.91 & \\
\hline & & & & & & 5.91 & -0.45 \\
\hline 4312.88 & Ti II & 1.18 & -1.160 & c2004 & 93 & 4.14 & \\
\hline 4399.77 & Ti II & 1.24 & -1.290 & c2004 & 86 & 4.25 & \\
\hline 4443.81 & Ti II & 1.08 & -0.700 & c2004 & 140 & 3.93 & \\
\hline 4450.49 & Ti II & 1.08 & -1.450 & n1996 & 59 & 4.08 & \\
\hline 4468.50 & Ti II & 1.13 & -0.600 & c2004 & 134 & 3.82 & \\
\hline 4501.28 & Ti II & 1.12 & -0.760 & c2004 & 122 & 3.90 & \\
\hline 4563.77 & Ti II & 1.22 & -0.820 & c2004 & 132 & 4.09 & \\
\hline \multirow[t]{3}{*}{4571.98} & Ti II & 1.57 & -0.340 & c2004 & 152 & 3.97 & \\
\hline & & & & & & 4.02 & -1.00 \\
\hline & & & & & & \pm 0.14 & \\
\hline 4252.63 & Cr II & 3.86 & -1.810 & sl90 & 51 & 5.48 & \\
\hline 4588.20 & Cr II & 4.07 & -0.630 & s190 & 142 & 5.14 & \\
\hline 4592.06 & Cr II & 4.07 & -1.230 & s190 & 112 & 5.52 & \\
\hline 4812.35 & Cr II & 3.86 & -1.960 & s190 & 52 & 5.63 & \\
\hline 4848.25 & $\mathrm{Cr}$ II & 3.86 & -1.150 & s190 & 120 & 5.36 & \\
\hline 4876.40 & $\mathrm{Cr}$ II & 3.85 & -1.450 & s190 & 105 & 5.56 & \\
\hline 5237.32 & Cr II & 4.07 & -1.160 & s190 & 152 & 5.73 & \\
\hline 5305.87 & Cr II & 3.83 & -1.790 & gs87 & 61 & 5.53 & \\
\hline 5308.43 & Cr II & 4.26 & -1.550 & gs87 & 64 & 5.60 & \\
\hline \multirow[t]{3}{*}{5313.59} & Cr II & 4.07 & -1.230 & gs87 & 83 & 5.31 & \\
\hline & & & & & & 5.48 & -0.19 \\
\hline & & & & & & \pm 0.17 & \\
\hline 4077.72 & Sr II & 0.00 & 0.170 & s2004 & 124 & 1.79 & \\
\hline \multirow[t]{2}{*}{4215.54} & Sr II & 0.00 & -0.170 & s2004 & 124 & 2.12 & \\
\hline & & & & & & 1.96 & -1.01 \\
\hline 4554.04 & Ba II & 0.00 & 0.170 & s2004 & 33 & 1.63 & \\
\hline \multirow[t]{2}{*}{4934.10} & Ba II & 0.00 & -0.160 & a2007 & 32 & 1.92 & \\
\hline & & & & & & 1.78 & -0.35 \\
\hline
\end{tabular}

References to Table 3: a2007: Aoki et al. (2007); c2004: Cohen et al. (2004); gs87: Gratton \& Sneden (1987); n1996: Norris et al. (1996); n2001: Norris et al. (2001); s190: Sigut \& Landstreet (1990); s2004: Sivarani et al. (2004); v93: Venn (1993); wsm: Wiese et al. (1969).

errors in the equivalent width measurements and the uncertainties in the stellar parameters, which were deduced by taking into account their standard errors. The related uncertainties due to the atmospheric parameters, in the derived abundances are: carbon, 0.2 ; nitrogen, 0.2 ; oxygen, 0.1 ; magnesium, 0.1 ; titanium, 0.1 ; chromiun, 0.2 ; barium, 0.2 ; and strontium, 0.2 . 


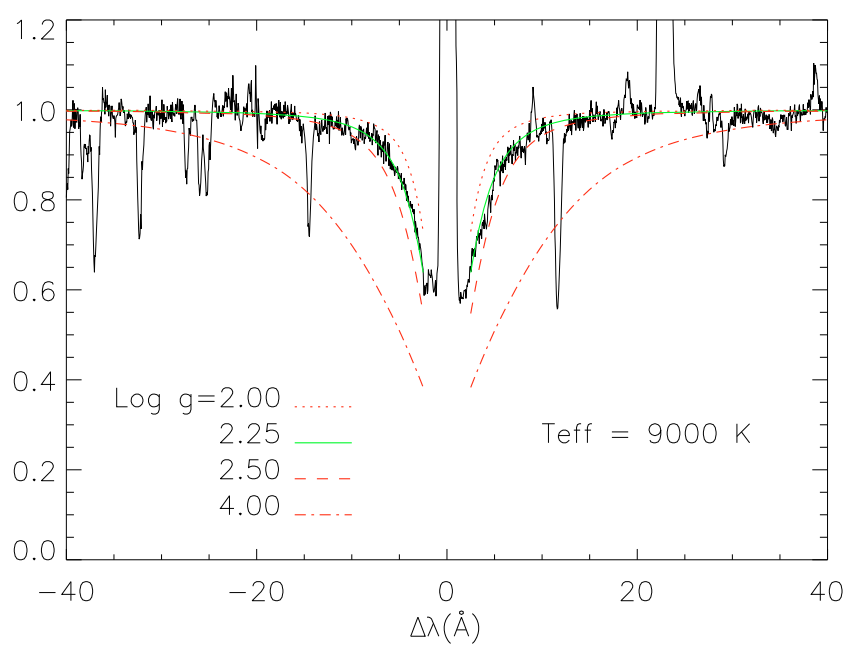

Fig. 4. Comparison between the observed spectrum of HD 149427 (solid black line) in the region of $\mathrm{H} \gamma$ and theoretical profiles computed for $T_{\text {eff }}=9000 \mathrm{~K}$ and $\log g=2.00,2.25,2.50$ and 4.00 (the different lines are identified in the figure). The best fit was obtained for $\log g=2.25$.

\section{Discussion}

\subsection{Stellar parameters and evolutionary tracks}

With the stellar parameters in hand, the primary star of HD 149427 can be located on a $\log g-\log T_{\text {eff }}$ diagram and compared with stellar evolutionary model tracks. Figure 5 (upper panel) shows the position of HD 149427 in the $\log g-\log T$ diagram, where model tracks were computed by Schaerer et al. (1993) for a solar metallicity. For comparison purposes, Fig. 5 also includes the position of HD 19993 which has a spectral type similar to that of HD 149427. The parameters derived for HD 149427 indicate a mass of $7 M_{\odot}$. The relation among temperature, gravity, mass, and luminosity is given by

$$
\log \left(L / L_{\odot}\right)=\log \left(M / M_{\odot}\right)-\log g+4 \log T_{\text {eff }}-10.61 .
$$

With the values of $T_{\text {eff }}=8700 \mathrm{~K}, \log g=2.3$, and $M / M_{\odot}=7$, the resulting luminosity is $\log \left(L / L_{\odot}\right)=3.69 \pm 0.32$. Figure 5 (lower panel) shows the position of HD 149427 and HD 19993 in the HR-diagram. Thus, HD 149427 is most likely to be an A3-A4 giant (luminosity class II).

The relation among temperature, gravity, mass, $V$ magnitude, and the interstellar absorption $\left(A_{V}\right)$ is given by:

$$
\begin{aligned}
\log r(\mathrm{kpc})= & \frac{1}{2}\left(\log \frac{M_{\star}}{M_{\odot}}+0.4\left(V-A_{V}\right)\right. \\
& \left.+4 \log T_{\mathrm{eff}}-\log g-16.5\right) .
\end{aligned}
$$

For the temperature, gravity, mass, above mentioned, $A_{V}=2.2$ (see above) and $V=12.68$ (Tylenda et al. 1992), Eq. (2) can be written, assuming no bolometric correction, as

$$
\log r(\mathrm{kpc})=\frac{1}{2}(4.295-\log g)
$$

and we obtain a distance $r=9.94 \pm 0.6 \mathrm{kpc}$ which is in good agreement with the value of $9 \mathrm{kpc}$ given by (Tajitsu \& Tamura 1998).

\subsection{Radius and luminosity of white dwarf}

Another piece of data constraining the distance to HD 149427 is provided by the white dwarf star in the binary system. Gutí1errez-Moreno \& Moreno (1998) derive $T_{\text {eff }}=105000 \mathrm{~K}$ for the white dwarf in HD 149427. The luminosity of the white dwarf can be obtained from

$$
\left(\frac{R^{*}}{d}\right)^{2}=\frac{F_{\lambda}^{\mathrm{c}}}{\frac{h c}{\lambda(\mathrm{H} \beta)}} \frac{c^{2}}{2 \pi}\left(\frac{h}{k T^{*}}\right)^{3} \frac{1}{G_{i}\left(T^{*}\right)} \frac{\alpha_{\mathrm{B}}\left(T_{\mathrm{e}}\right)}{\alpha_{\mathrm{eff}}\left(T_{\mathrm{e}}\right)}
$$

and

$L=4 \pi \sigma R^{* 2} T^{* 4}$

where $F_{\lambda}^{\mathrm{c}}$ is the observed $\mathrm{H} \beta$ flux corrected for interstellar reddening, $\alpha_{\mathrm{B}}\left(T_{\mathrm{e}}\right)$ and $\alpha_{\mathrm{eff}}\left(T_{\mathrm{e}}\right)$ are the total and effective recombination coefficients, respectively, for the line considered. $G_{i}\left(T^{*}\right)$ strongly depends on the radiation temperature and numerical values for $\mathrm{H}^{0}$ are given in Pottasch (1984). For $T_{\text {eff }}=105000 \mathrm{~K}, \alpha_{\mathrm{B}}\left(T_{\mathrm{e}}\right)$ and $\alpha_{\mathrm{eff}}\left(T_{\mathrm{e}}\right)$ depend on the nebular parameters. Adopting $T_{\mathrm{e}}=18000 \mathrm{~K}$ and $\log N_{\mathrm{e}}=5.0$ (Gutiérrez-Moreno \& Moreno 1998), $\alpha_{\mathrm{B}}\left(T_{\mathrm{e}}\right)$ and $\alpha_{\mathrm{eff}}\left(T_{\mathrm{e}}\right)$ have values of $2.6 \times 10^{-13} \mathrm{~cm}^{3} \mathrm{~s}^{-1}$ and $3.0 \times 10^{-14} \mathrm{~cm}^{3} \mathrm{~s}^{-1}$, respectively. The observed $\mathrm{H} \beta$ flux did not change in the past few years and from our low-resolution spectrum we infer $6.6 \times 10^{-12} \mathrm{erg} \mathrm{cm}^{-2} \mathrm{~s}^{-1}$ corrected for $A_{V}=2.2$. Finally, the radius of the white dwarf at $10 \mathrm{kpc}$ is $0.14 R_{\odot}$ and the luminosity is approximately $2000 L_{\odot}$. In the $\log L-\log T_{\text {eff }}$ plane, this white dwarf has a post-AGB age of $\sim 20000 \mathrm{yr}$ for a mass of $0.57 M_{\odot}$ (Schönberner 1983). This age is older than expected from the young PN characteristics of PC 11. Nevertheless, the value of $T_{\text {eff }}$ for the white dwarf is somewhat uncertain (see Gutiérrez-Moreno \& Moreno 1998). A higher $T_{\text {eff }}$ would imply a higher mass for the white dwarf and, hence, a younger post-AGB age (see Blöcker \& Schönberner 1990). A more precise determination of $T_{\text {eff }}$ for the hot star is necessary to obtain a more accurate estimate of its the age.

\subsection{Mass and radius of the ionized nebula}

The radius and the mass of the ionized nebula are determined using the parameters obtained in the previous sections. The radius can be obtained from the $\mathrm{H} \beta$ flux corrected for reddening. We assume that $N_{\mathrm{e}}$ is approximately equal to $N_{\mathrm{p}}$, and then,

$F^{\mathrm{c}}(\mathrm{H} \beta)=\frac{1}{4 \pi d^{2}} \frac{h c}{\lambda_{\mathrm{H} \beta}} \int_{0}^{R} N_{\mathrm{e}} N_{\mathrm{p}} \alpha_{\mathrm{eff}}\left(T_{\mathrm{e}}\right) \mathrm{d} V$,

where $d$ is the distance to the HD 149427. Using the values of $F_{\mathrm{H} \beta}^{\mathrm{c}}, N_{\mathrm{e}}$ and $\alpha_{\mathrm{eff}}\left(T_{\mathrm{e}}\right)$ calculated in the previous section, we find $R \approx 0.01$ pc or $\theta \approx 0.2 \operatorname{arcsecs}$ at $10 \mathrm{kpc}$. The ionized mass $\left(M_{\mathrm{i}}\right)$ is derived using the simple approximation of spherical symmetry

$M_{\mathrm{i}}=\frac{4 \pi}{3} R^{3} N_{\mathrm{e}} m_{\mathrm{H}}$

where $m_{\mathrm{H}}$ is the mass of the hydrogen atom. The value obtained for $M_{\mathrm{i}}$ is $\approx 0.01 M_{\odot}$, a value consistent with that expected for a PN with $N_{\mathrm{e}}=10^{5} \mathrm{~cm}^{-3}$ (Pottasch 1984, p. 113).

HST images of HD 149427 show that the nebula consists of two regions: a very compact, elongated inner region, and an extended, round, outer shell (or halo) (Parthasarathy et al. 2000). The images also show that most of the emission is generated in the inner region, while the contribution of the outer shell to the total emission is very small. Parthasarathy et al. (2000) quoted 


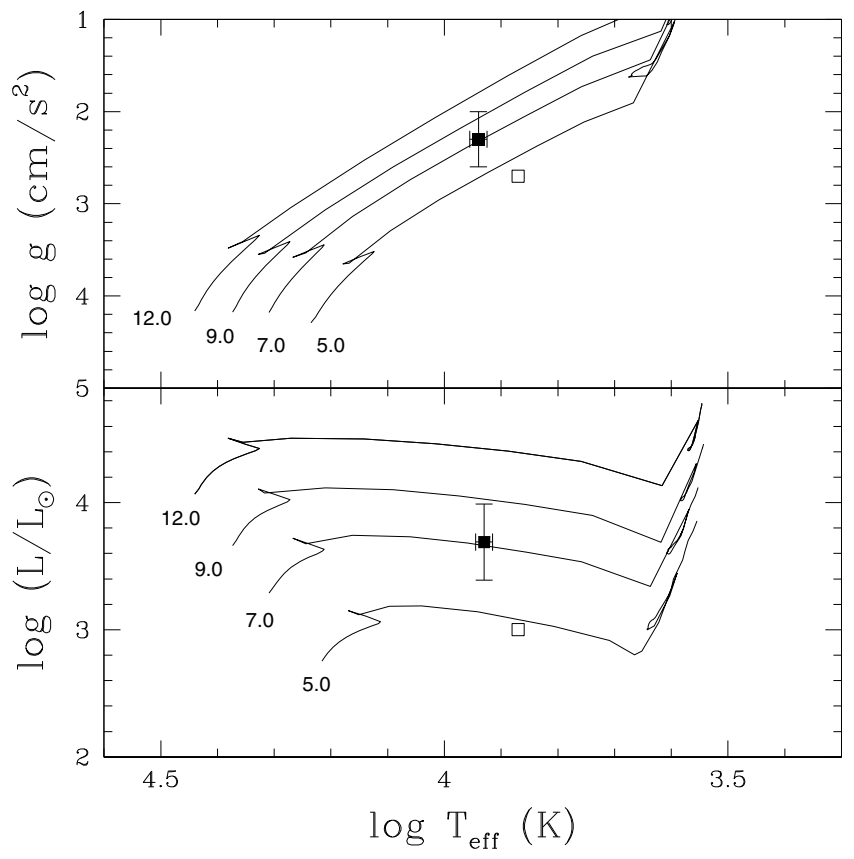

Fig. 5. The positions of HD 149427 (filled square) and the A7-A8 giant HD 19993 (open square) in both the $\log g-\log T_{\text {eff }}$ diagram (upper panel) and the HR-diagram (lower panel) along with stellar model evolutionary tracks from Scharer et al. (1993). Evolutionary tracks are given in units of solar mass. Data from HD 19993 ware taken from Miroshinichenko et al. (2003).

a diameter of $<0.8^{\prime \prime}$ for the inner region. However, a reinspection of the HST images indicates that a diameter of $\simeq 0.5^{\prime \prime}$ is more appropiate for this region. This size coincides quite well with the size of $0.4^{\prime \prime}$ for the ionized nebula derived from the $\mathrm{H} \beta$ flux (see above). Given the widely different contributions of the two regions to the total flux, the $\mathrm{H} \beta$ emission in our spectra should mainly originate in the bright inner region. The coincidence in sizes also provides support for a distance of $\simeq 10 \mathrm{kpc}$ to HD 149427. At a galactic latitude of 5 degrees, considering the distance determined above, HD 149427 should be almost 900 pc above the Galactic plane.

\subsection{The abundance pattern}

In Fig. 6, the CNO abundances of the A-type star in HD 149427 are compared with those of other evolved objects in the diagram $\mathrm{O} / \mathrm{N}$ versus $\mathrm{C} / \mathrm{N}$, including Population I supergiants (Luck \& Bond 1986; Luck \& Lambert 1985), PNe (Kingsburgh \& Barlow 1994), symbiotic stars (Nussbaumer et al. 1988), and post-AGB stars. The thick line represents the ratio $\mathrm{C} / \mathrm{O}=1.0$. In this figure, two types of post-AGB stars can be distinguished, those that display the $21 \mu \mathrm{m}$ circunstellar feature and show an overabundance of carbon and the elements created by slow neutron capture reactions (van Winckel \& Reyniers 2000), and those that do not exhibt overabundances in carbon nor in the elements created by slow neutron capture reactions (Luck et al. 1990; van Winckel 1997; Pereira 2004).

Figure 6 clearly shows that Pop I supergiants show traces of nitrogen enrichment due to $\mathrm{CNO}$-cycling. PNe are found on both sides of the $\mathrm{C} / \mathrm{O}=1$ line. Some PNe seem to display only CNO processed material and are in the same position of the diagram as Pop I supergiants, while those on the other side of the $\mathrm{C} / \mathrm{O}$ line show enhancement of carbon due to He burning. Post-AGB stars with the $21 \mu \mathrm{m}$ circunstellar feature lie in the same location as

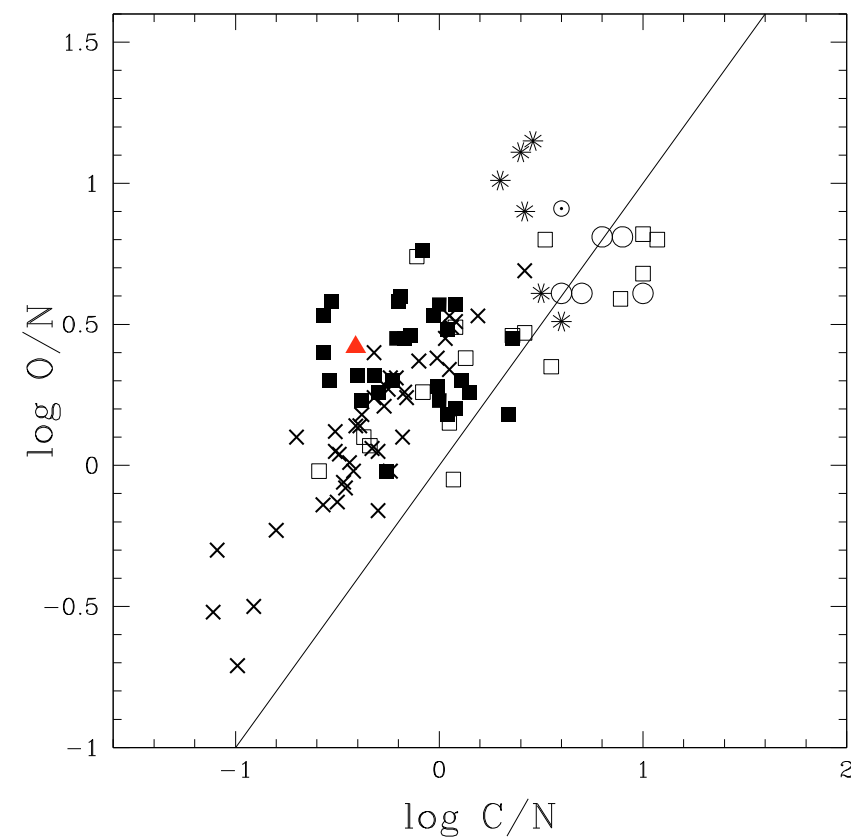

Fig. 6. $\mathrm{O} / \mathrm{N}$ abundance ratio against $\mathrm{C} / \mathrm{N}$ ratio for post- $\mathrm{AGB}$ stars $\mathrm{PNe}$, Pop. I supergiants, symbiotic stars and HD 149427. Open squares: PNe; diagonal cross points: Pop. I supergiants; open circles: post-AGB stars that display the $21 \mu \mathrm{m}$ circunstellar feature; asterisks: oxygen-rich postAGB stars; filled square points: symbiotic stars; HD 149427: filled red triangle.

PNe with $\mathrm{C} / \mathrm{O} \geq 1.0$. The position of HD 149427 in Fig. 6 is indicative of nitrogen enrichment due to $\mathrm{CNO}$-cycling. The low $\mathrm{C} / \mathrm{O}$ ratio (0.15), which is lower than in the Sun $\left(\mathrm{C} / \mathrm{O}_{\odot}=0.51\right)$, indicates that helium burning is not yet taking place in the interior of HD 149427.

The mean value of $\alpha$-elements $(\mathrm{Mg}, \mathrm{Si}, \mathrm{Ca}$ and $\mathrm{Ti})$ given by the ratio $[\alpha / \mathrm{Fe}]=-0.34$ which is lower than observed for disk stars of the same metallicity (Gratton \& Sneden 1987). Chromiun, an element that belongs to the iron-group, is correlated with the iron abundance, and is slightly underabundant.

The heavy-element abundance in the A-type star, as given by the $[\mathrm{Sr} / \mathrm{H}]$ and $[\mathrm{Ba} / \mathrm{H}]$ ratios, show no enrichment of these elements which are created by slow neutron capture reactions.

\section{5. $H D 149427$ in the diagnostic diagram $5007 / H \beta$ versus $4363 / \mathrm{H \gamma}$}

HD 149427 has been classified as a low-excitation PN (Parthasarathy et al. 2000) and also as a D'-type symbiotic star based on its infrared colors (Allen 1982). Since all D'-type symbiotics have F-G spectral types (Schmid \& Nussbaumer 1993), the A-F continuum of HD 149427 is also indicative of the symbiotic nature of this object. Figure 7 compares the low-resolution spectrum of HD 149427 with the spectra of HD 330036 and Hen 3-1312. It is worth noting that these three objects have similar emission and continuum spectra but different natures. HD 330036 is a well known D'-type symbiotic star (Schmid \& Nussbaumer 1993; Pereira et al. 2005), while Hen 3-1312 is another peculiar, low-excitation PN hosting a post-AGB star (Pereira 2004). The evolutionary status of the cool component is not the only difference among the three objects. The relative strengths of the [O III] $\lambda 4363$ emission lines also differ. Therefore, in the diagram $5007 / \mathrm{H} \beta$ versus $4363 / \mathrm{H} \gamma$, these three objects occupy different positions. In Fig. 8 we show such a 




Fig. 7. Optical spectrum of HD 149427 between $3500 \AA$ and $7500 \AA$ compared to that of the $\mathrm{D}^{\prime}$-type symbiotic star HD 330036 and the peculiar PN Hen 3-1312. Note the strength of the [O III] $\lambda 4363$ emission relative to the $\mathrm{H} \gamma$ emission in these three objects. The $\mathrm{H} \alpha$ emission line in Hen 3-1312 is saturated. The units of flux $\operatorname{are~erg} / \mathrm{cm}^{2} / \mathrm{s} / \AA$.

diagram for PNe, symbiotic stars, Hen 3-1312, and HD 149427. HD 149427 is located near the region occupied by PNe but close to the position of the symbiotic star H1-36, which, combined with the A-F continuum of the central star causes confusion when classifying HD 149427.

\section{Conclusions}

We have analyzed the emission and the absorption spectrum of the peculiar PN HD 149427 using low- and high-resolution spectroscopy. The main conclusions of this work can be summarized as follows:

1. The stellar parameters derived for the A-star in the binary central star are: $T_{\text {eff }}=8600 \mathrm{~K}, \log g=2.3$, and $V_{\text {rot }}=$ $30 \mathrm{~km} \mathrm{~s}^{-1}$. These parameters correspond to an A(3-4)II star with a mass of $\simeq 7 M_{\odot}$ and a luminosity of $\simeq 5000 L_{\odot}$, located at a distance of $\simeq 10 \mathrm{kpc}$.

2. The A-star exhibits a mild deficiency of iron. Barium and strontium, monitors of s-process nucleosynthesis, do not display any degree of enrichment. The other elements are underabundant compared to those of Sun. As for the light elements, nitrogen has been enhanced by CNO-cycling, but carbon is not enriched, which means that helium burning has not yet started. The general abundance pattern is similar to the A(7-8)II giant analyzed by Miroshnichenko et al. (2003).

3 . From the position of HD 149427 in the $\lambda 5007 / \mathrm{H} \beta-$ $\lambda 4363 / \mathrm{H} \gamma$ diagram, we show that its position coincided neither with that of PNe nor that of symbiotic stars. This is because of to electron density observed in HD 149427, which is higher than typical electron densities in PNe, but not as high as observed in D- and $\mathrm{D}^{\prime}$-type symbiotics.

Acknowledgements. We are grateful to our referee, R. Méndez, for his comments and critical readding of the manuscript. This work has been supported



Fig. 8. Position of PNe, D'-, D-, and S-type symbiotics, the young PN PM 1-322, HD 149427, and Hen 3-1312 in the $\lambda 5007 / \mathrm{H} \beta-$ $\lambda 4363 / \mathrm{H} \gamma$ plane. Open squares: PNe (Kingsburgh \& Barlow 1994); filled squares: D- and $\mathrm{D}^{\prime}$-type symbiotics (Schmid \& Nussbaumer 1993; Gutiérrez-Moreno \& Moreno 1996; Schmid \& Schild 1990; Pereira 1995; Pereira et al. 1998;) filled circles: S-type symbiotics (Pereira 1995; Gutiérrez-Moreno et al. 1999; Schmid \& Schild 1990); cross: PM 1-322 (Pereira \& Miranda 2005); red asterisk: HD 149427 (this work); red triangle: Hen 3-1312 (Pereira 2004).

partially by grant AYA2008-01934 of the Spanish MICINN (co-funded by FEDER funds), and by grant FGM-1747 of the Junta de Andalucía.

\section{References}

Acker, A., Ochsenbein, F., Stemholm, B., et al. 1992, Strasbourg-ESO Catalogue of Galactic Planetary Nebulae

Allen, D. A. 1982, in The Nature of Symbiotic Stars, ed. M. Friedjung, \& R. Viotti (D. Reidel Publishing Co), IAU Coll., 70, 27

Aoki, W., Beers, T. C., Christlieb, N., et al. 2007, ApJ, 655, 492

Blöcker, T., \& Schönberner, D. 1990, A\&A, 240, L11

Bond, H. 2000, in From Origins to Microstructures, Binarity of Central Stars of Planetary Nebulae, ed. J. H. Kastner, N. Soker, \& S. Rapapport, ASP Ser., 199,115

Bond, H. E., Pollacco, D. L., \& Webbink, R. F. 2003, AJ, 125, 260

Cohen, J. G., Christlieb, N., McWilliam, A., et al. 2004, ApJ, 612, 1107

Daflon, S., Cunha, K., \& Butler, K. 2004, ApJ, 604, 362

de Marco, O. 2009, PASP, 121, 316

Gratton, R. G., \& Sneden, C. 1987, A\&A, 178, 179

Gutiérrez-Moreno, A., \& Moreno, H. 1996, PASP, 108, 972

Gutiérrez-Moreno, A., \& Moreno, H. 1998, PASP, 110, 458

Gutiérrez-Moreno, A., Moreno, H., \& Costa, E. 1999, PASP, 111, 571

Hamuy, M., Suntzeff, N. B., Heathcote, S. R., et al. 1994, PASP, 106, 566

Hummer, D. G., \& Storey, P. J. 1987, MNRAS, 224, 801

Kaufer, A., Stahl, O., Tubbesing, S., et al. 1999, The Mesenger, 95, 8

Kingsburgh, R. L., \& Barlow, M. J. 1994, MNRAS, 271, 257

Kurucz, R. L. 1993, CD-ROM 13, Atlas9 Stellar Atmosphere Programs and

$2 \mathrm{~km} \mathrm{~s}^{-1}$ Grid (Cambridge: Smithosian Astrophys. Obs.)

Lambert, D. L., Heath, J. E., Lemke, M., \& Drake, J. 1996, ApJS, 103, 183

Luck, R. E., \& Lambert, D., 1985, ApJ, 298, 782

Luck, R. E., \& Bond, H. E. 1989, ApJS, 71, 559

Luck, R. E., Bond, H. E., \& Lambert, D. L. 1990, ApJ, 357, 188

Lutz, J. 1977, A\&A, 60, 93

McWilliam, A., Preston, G. W., Sneden, C., \& Shectman, S. 1995, AJ, 109, 2736 Miszalski, B., Acker, A., Moffat, A. F. J., Parker, Q. A., \& Udalski, A. 2008, A\&A, 488, L79

Méndez, R. H. 1978, MNRAS, 185, 647 
C. B. Pereira et al.: Spectroscopic analysis of the peculiar planetary nebula HD 149427

Méndez, R. H. 1989, Binary and intrinsic variability in central stars of PN, in Planetary Nebulae, ed. S. Torres-Peimbert, IAU Symp., 131, 261

Miroshnichenko, A. S., Kusakin, A. V., \& Bjorkman, K. S. 2003, A\&A, 412, 219

Norris, J. E., Ryan, S. G., \& Beers, T. C. 1996, ApJS, 107, 391

Norris, J. E., Ryan, S. G., \& Beers, T. C. 2001, ApJ, 561, 1034

Nussbaumer, H., Schmid, H. M., Vogel, M., \& Schild, H. 1988, A\&A, 198, 179

Oke, J. B. 1974, ApJS, 27, 21

Parthasarathy, M., García-Lario, P., Pottasch, S. R., de Martino, D., \& Surendiranath, R. 2000, A\&A, 355, 720

Pereira, C. B. 1995 , A\&AS, 111, 471

Pereira, C. B. 2004, A\&A, 413, 1009

Pereira, C. B., \& Miranda, L. F. 2005, A\&A, 433, 579

Pereira, C. B., \& Miranda, L. F. 2007, A\&A, 467, 1249

Pereira, C. B., Landaberry, S. J. C., \& Junqueira, S. 1998, A\&A, 333, 658

Pereira, C. B., Smith, V. V., \& Cunha, K. 2005, A\&A, 429, 993

Pereira, C. B., Miranda, L. F., Smith, V. V., \& Cunha, K. 2008, A\&A, 477, 535

Pottasch, S. R. 1984, Planetary Nebulae (Dordrecht: D.R. Reidel)

Royer, F., Grenier, S., Baylac, M. O., Gómez, A. E., \& Zorec, J. 2002, A\&A, 393, 897
Sanduleak, N., \& Stenphenson, C. B. 1972, PASP, 84, 816

Schaerer, D., Charbonnel, C., Meynet, G., Maeder, A., \& Schaller, G. 1993, A\&AS, 102, 339

Schmid, H. M., \& Nussbaumer, H. 1993, A\&A, 268, 159

Schmid, H. M., \& Schild, H. 1990, MNRAS, 246, 84

Schönberner, D. 1983, ApJ, 272, 708

Seaton, M. J. 1979, MNRAS, 187, 73

Shen, Z.-X., Liu, X.-W., \& Danziger, I. J. 2004, A\&A, 422, 563

Sigut, T. A. A., \& Landstreet, J. D. 1990, MNRAS, 247, 611

Sivarani, T., Bonifacio, P., Molaro, P., et al. 2004, A\&A, 413, 1073

Sneden, C. 1973, Ph.D. Thesis, Univ. of Texas

Tajitsu, A., \& Tamura, S. 1998, AJ, 115, 1989

Thévenin, F., \& Jasniewicz, G. 1997, A\&A, 320, 913

Tylenda, R., Acker, A., Stenholm, B., \& Koeppen, J. 1992, A\&AS, 95, 337

Van Winckel, H. 1997, A\&A, 319, 561

Van Winckel, H., \& Reyniers, M. 2000, A\&A, 354, 135

Venn, K. A. 1993, ApJ, 414, 316

Webster, L. B. 1966, PASP, 78, 136

Wiese, W. L., Smith, M. W., \& Miles, B. M. 1969, NBS Ref. Data. Ser. 